\title{
Frequency discrimination of complex periodic tones*
}

\author{
L. L. FETH \\ University of Oklahoma Health Sciences Center. Oklahoma City, Oklahoma 73190
}

\begin{abstract}
When the components of a two-tone periodic complex differ slightly in amplitude and frequency, the pitch is shifted toward the more intense tone. This well-known phenomenon has been explained by differences in the instantaneous frequency functions of the complex tones. In this experiment, three listeners were asked to discriminate between complementary pairs of two-component complex tones. The results indicate that discriminability may depend upon differences in the envelope-weighted instantaneous frequency functions of the two-tone complexes.
\end{abstract}

More than 100 years ago, Helmholtz "explained" the pitch shifts that were perceived when a tone of given frequency and amplitude was sounded simultaneously with another which differed slightly in both frequency and amplitude (Helmholtz, 1954; von Békésy, 1960; Jeffress, 1968). He observed that the combination $\left(f_{o}, I_{o}+\Delta I\right)$, $\left(f_{o}+\Delta f, I_{0}\right)$ was lower in pitch than $\left(f_{0}, I_{0}\right),\left(f_{o}+\Delta f, I_{0}+\Delta I\right)$. That is, the pitch seemed to shift toward the more intense tone.

Spectral representations of these complex periodic tones are shown in the left-hand column of Fig. 1. In the top row, Tone $A$ is at Frequency $f_{o}$ and Level $\left(I_{o}+\Delta I\right)$. Tone $B$ at $\left(f_{o}+\Delta f\right)$ is at Level $I_{o}$. In the lower row, Tone $A$, still at $f_{o}$, has been reduced in level to $I_{o}$; Tone $B$, at $\left(f_{o}+\Delta f\right)$, has been raised to Level $\left(I_{o}+\Delta I\right)$. We may summarize Helmholtz's observation by stating that for a number of combinations of $\Delta \mathrm{I}$ and $\Delta \mathrm{f}$, the complex in the upper row will sound lower in pitch than the one represented in the bottom row.

Helmholtz explained this phenomenon after calculating the instantaneous frequency function for each combination. Jeffress gives a similar derivation. We will briefly outline the derivation here, with the help of Fig. 1, because the results are essential for the extension of this work that we propose. In an equation, the two-tone combination can be written:

$$
y(t)=a \cos \left(2 \pi f_{o} t\right)+b \cos \left[2 \pi\left(f_{o}+\Delta f\right) t\right] .
$$

Here $\Delta f$ is always a positive number, and three situations are possible with respect to the pressure amplitudes, a and b. Clearly, either a is greater than b, or less than b, or equal to $b$. Some trigonometric manipulations allow us to put Eq. 1 into the form:

$$
y(t)=E(t) \cos \left[2 \pi f_{o} t+\phi(t)\right] .
$$

Here $E(t)$ is the envelope function which follows the slowly varying peaks in the instantaneous value of the complex tone. It is given by:

\footnotetext{
*The data for this experiment were collected while the author was a NSF postdocotral fellow with the Department of Psychology, University of California, San Diego.
}

$$
E(t)=a^{2}+2 a b \cos 2 \pi \Delta f t+b^{2} .
$$

The function $\phi(t)$ expresses the instantaneous value of the phase of the complex with Tone $A$ as a reference. We obtain the instantaneous frequency function by taking a time derivative of $\phi(t), f(t)=d \phi(t) / d t$. This gives us,

$$
f(t)=f_{o}+\frac{a b \Delta f \cos 2 \pi \Delta f t+b^{2} \Delta f}{a^{2}+2 a b \cos 2 \pi \Delta f t+b^{2}} .
$$

When $a=b$, the familiar auditory beat phenomenon is perceived (see, for example, Licklider, 1951). The amplitude of the complex tone waxes and wanes from a maximum value of $2 \mathrm{a}$ to a minimum of zero, $1 / \Delta \mathrm{f}$ times per second. Of course, when $\Delta \mathrm{f}$ becomes large, the percept becomes a smooth complex tone with no noticeable amplitude fluctuations.

Our interest lies in the two cases where there are differences in the amplitudes of the component tones. Consider, first, the amplitude fluctuations for a given pair of two-tone signals. For given values of $a$ and $b$ with $a \neq b$, either (1) $a>b$, or (2) $a<b$. Examination of Eq. 3 leads us to expect no difference in the envelope function for the pair since $a$ and $b$ are represented symmetrically. The center portion of Fig. 1 illustrates typical enveloped functions for a complex pair. Peak amplitude varies from a maximum of $(a+b)$ to $a$ minimum of $(a-b), 1 / \Delta f$ times per second. This function can be observed on an oscilloscope if the sweep time is on the order of $1 / \Delta \mathrm{f}$.

Inspection of Eq. 4 reveals asymmetries which lead to different functions $f(t)$ for each two-tone complex in a given pair. The right-hand portion of Fig. 1 gives a typical example. Here the abscissa is time, as in the center portion, but the ordinate is now frequency. Thus, in the upper row, the instantaneous value of $f(t)$ varies periodically from a value just below $\left[f_{o}+\left(f_{o}+\Delta f\right)\right] / 2$ to frequencies even lower than $f_{o}$. In the lower row, the instantaneous frequency function is the mirror image of the one above. Now the flat portion is just above the average of $f_{o}$ and $\left(f_{o}+\Delta f\right)$, and the peaks extend well above $\left(f_{o}+\Delta f\right)$. The equations indicate that the peaks in 
SPECTRUM
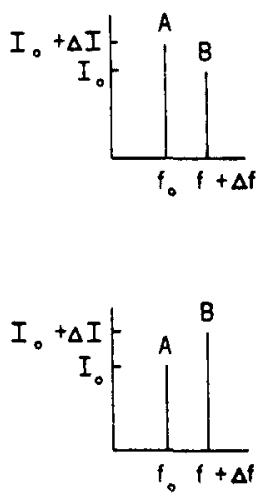

ENVELOPE

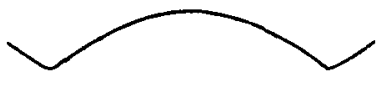

岁

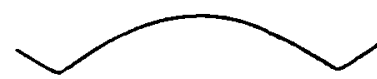

TIME
INSTANTANEOUS FREQUENCY

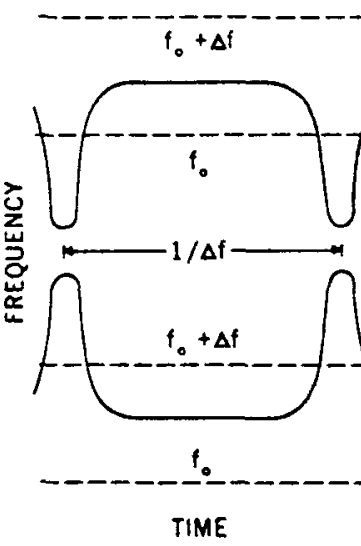

Fig. 1. Spectrum, envelope, and instantaneous frequency functions for both members of a set of two-component periodic tones. $f(t)$ always occur at the minima of the envelope function. Also, as the difference between $a$ and $b$ is reduced, the instantaneous frequency function "spends more time" near the midline, but moves to more extreme values for the brief time that it deviates from that value.

Previous authors have reasoned that the pitch difference between the members of a complex-tone pair must be due to differences in their instantaneous frequency functions. In fact, Jeffress demonstrated this convincingly by listening only to portions of the signal near envelope minima. Apparently, none of the previous investigators attempted to quantify the differential pitch sensation produced by these tones.

\section{METHODS}

\section{Listeners}

Three listeners with normal hearing were asked to discriminate between pairs of complex tones in a standard two-interval, forced-choice (2IFC) paradigm. These listeners were undergraduates at the University of California, San Diego, who were paid $\$ 1.75$ per hour for their participation.

\section{Apparatus}

Figure 2 shows a block diagram of the apparatus used to generate two-component complex tones. Two oscillators, set to produce $f_{o}$ and $\left(f_{o}+\Delta f\right)$, supply the basic signals. To establish the small differences in amplitude, an attenuated version of each signal is added to itself. The other signal is also added at this point; thus, very precise three-input signal summers are necessary for this experiment.

Each of the two electronic switches presents a complex tone at the appropriate time. Beyond the gates, the signal pathways are combined for monaural presentation, although the gates are not turned on simultaneously.

\section{Procedure}

Using the 2IFC paradigm, frequency and amplitude differences $(\Delta f$ and $\Delta \mathrm{I})$ remained fixed for a block of 50 trials, and the percentage of correct discriminations, $P(C)$, was determined for each listener. For a given amplitude difference $(.2, .5$, or $1 \mathrm{~dB})$, the frequency difference was changed from block to block to obtain a psychometric function. These display $P(C)$ as a function of $\Delta f$ with $\Delta I$ as the parameter. Listeners were given extensive practice at all conditions before 200 trials at each $\Delta \mathrm{f}-\Delta \mathrm{I}$ combination were recorded for inclusion in the data. For this experiment, $f_{o}$ was $1,000 \mathrm{~Hz}, I_{o}$ was $70 \mathrm{~dB}$ SPL. Signals were gated on for $200 \mathrm{msec}$ and randomly assigned to the observation intervals.

In addition, simple frequency discrimination data $\left(f_{0}, I_{0}\right)$ vs

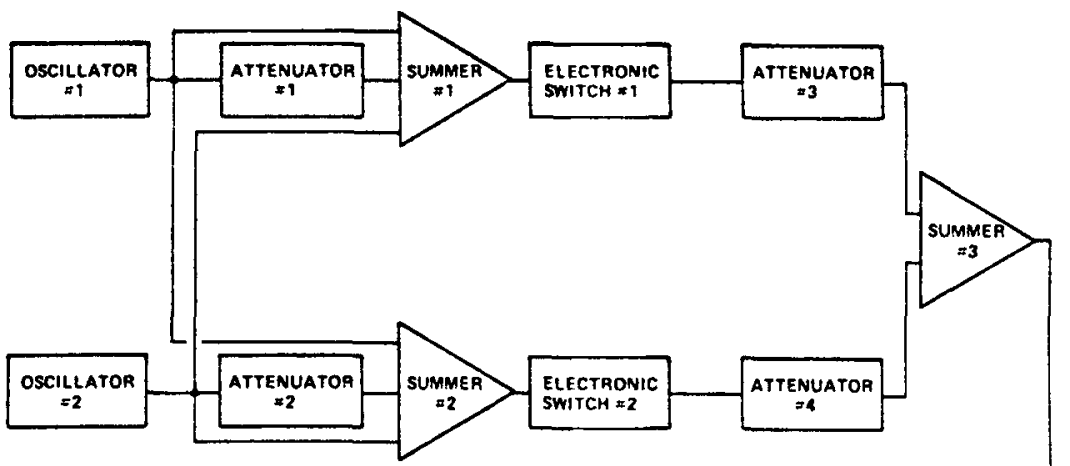

Fig. 2. A block dingram of the apparatus needed to generate two-component complex tones. 
$\left(f_{o}+\Delta f, I_{o}\right)$ were obtained for each listener. All experimental conditions were the same as for the two-tone discriminations.

\section{RESULTS}

The results of this experiment are displayed as psychometric functions for three listeners with normal hearing (Fig. 3). The open symbols represent the "raw" data percentage of correct discriminations, $P(C)$ in $21 F C$, as a function of $\Delta f$. The parameter is $\Delta I$. Each symbol represents 200 observations by each listener at a given $\Delta \mathrm{f}-\Delta \mathrm{l}$ combination. Also shown in the figure are the listeners' psychometric functions for simple (one tone) frequency discrimination (* symbol). For each listener, the (simple) frequency discrimination function lies to the left of his psychometric functions for the complex tones.

For single-frequency tones, small differences in pitch are monotonically related to differences in frequency (within the octave). For two-tone complex pairs, pitch differences also seem to be due to frequency differences; however, frequency varies periodically in the two-tone complexes. Let us assume, for the moment, that the auditory system processes single-frequency tones and two-tone complexes in the same way. Since frequency varies in the complex tone, let's assume that the discrimination is based upon a difference in average frequency. That is, suppose we calculate an average frequency for each of the two-tone complexes in a pair. Then our listener's ability to discriminate one member of the pair from the other should be monotonically related to the difference between these average frequencies. Since the simple frequency-discrimination psychometric function represents a limitation on our listener's performance, we should be able to collapse the families of psychometric functions shown in Fig. 3 onto the frequency discrimination function. To do this, we need only plot $P(C)$ as a function of the difference in averaged frequencies for each two-tone complex pair. Our problem is to find the appropriate averaging process.

Simple averaging procedures fail to produce the desired transformation. That is, a weighting such as $\left[a f_{o}+b\left(f_{o}+\Delta f\right)\right] /(a+b)$ for one complex tone, and $\left[b f_{o}+a\left(f_{o}+\Delta f\right)\right] /(a+b)$ for its mate predicts differences that are far too small. A better guess is the difference in time-averaged instantaneous frequency for the complex signals. Here, too, we encounter problems due to a curious property of these complex signals. Cherry and Phillips (1961) show that the time average of the instantaneous frequency function, $\hat{\mathrm{t}}(t)$, for two-tone complexes such as ours can take on only one of three unique values. If, in our Eq. $1, a>b$, then $f(t)$ equals $f_{0}$. If $a<b$, then $\hat{f}(t)=\left(f_{0}+\Delta f\right)$. If $a=b$, then $\hat{f}(t)=$ $\left[f_{0}+\left(f_{o}+\Delta f\right)\right] / 2$. This result indicates that the difference in $\mathrm{i}(\mathrm{t})$ for every complex pair would be equal to $\Delta f$ and independent of $\Delta \mathrm{I}$. Inspection of the psychometric functions in Fig. 3 shows that this prediction would be incorrect.
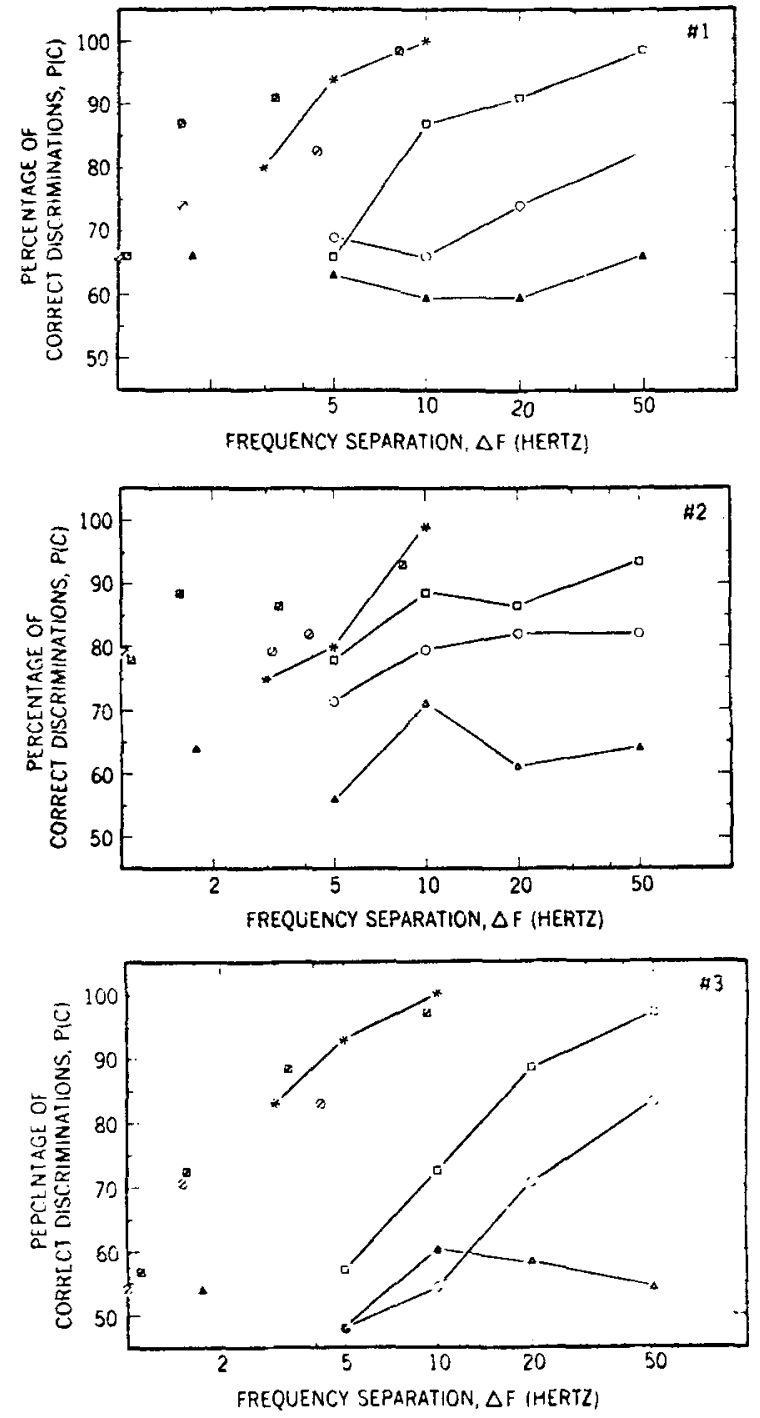

Fig. 3. Percentage of correct discriminations, for three listeners, as a function of the frequency separation of the two-component complex. The parameter is the intensity difference: $1.0 \mathrm{~dB}, \square ; 0.5 \mathrm{~dB}, \circ$; or $0.2 \mathrm{~dB}, \triangle$. Each listener's psychometric function for simple frequency discrimination is also given (*). Symbols with diagonal slashes represent differences in the weighted-average of the instantaneous frequency of corresponding data points (see text).

Let us briefly reconsider these complex tones. In addition to the periodic frequency modulations, there are simultaneous amplitude variations that are represented by the envelope function, $E(t)$. Suppose that the amplitude fluctuation has the effect of lending differential weights to concomitant values of instantaneous frequency. That is, suppose that the values of the instantaneous frequency near envelope maxima contribute proportionately more to the overall average than those values that occur near the minima. We then may calculate weighted averages of instantaneous frequency for each of the complex tones. If our listeners 
are basing their judgments on such weighted averages of instantaneous frequency, then their $P(c)$ score for a given difference should correspond with their performance in the simple frequency discrimination task.

An example from the data in Fig. 3 will illustrate this point. For Listener 3 , we plot a square symbol at $\Delta \mathrm{f}=$ $20 \mathrm{~Hz}, \mathrm{P}(\mathrm{C})=89 \%$. Thus, he is able to distinguish between the complex tones (1) $(1,000 \mathrm{~Hz}, 71 \mathrm{~dB})$, $(1,020 \mathrm{~Hz}, 70 \mathrm{~dB})$ and $(2)(1,000 \mathrm{~Hz}, 70 \mathrm{~dB}),(1,020 \mathrm{~Hz}$, $71 \mathrm{~dB}) 89 \%$ of the time. When we calculate the envelope-weighted average of the instantaneous frequency function for each complex tone, we get $1,007.59 \mathrm{~Hz}$ and $1,012.41 \mathrm{~Hz}$, respectively. The difference is $4.82 \mathrm{~Hz}$. Notice that this listener performs at a 93\% level when discriminating between two single-frequency tones with a $5-\mathrm{Hz}$ separation.

A similar transformation can be applied to every data point in Fig. 3. Essentially, this has been done, with the transformed data represented by symbols of the same shape but with a single line through them. (Thus, a square, $\square$, becomes $\square$, etc.).

One further refinement was attempted. The transformed points seemed to fit the simple discrimination function better when an envelope threshold was imposed. This is equivalent to giving a zero weight to the instantaneous frequency value at the envelope minima. It is apparent from an inspection of Fig. 3 that none of the fits are perfect. The psychometric functions for discrimination of single-frequency tones are represented by only three points. With so few degrees of freedom, curve fitting seemed imprudent. Obviously, the transformations produced the best fit for Listener 3 and a reasonable one for Listener 1. Listener 2 poses a problem. At $\Delta f$ of $10 \mathrm{~Hz}$, his performance exceeds that of the other listeners; however, at greater $\Delta f$, he seems to reach an asymptote or decline slightly. Simple frequency discriminations also differ for Listener 2. As a result, most of the transformed data points fall to the left of, and above, his simple frequency discrimination function.

\section{DISCUSSION}

We have speculated that the basis for discriminations between two-component periodic tones might lie in the listener's ability to perceive a weighted average of the instantaneous frequencies. The performance of two of our three listeners tends to support this model, although this preliminary study contains too little data to either accept or reject it at this time.

This work should be extended in several ways. Obviously, complex periodic tones containing three or more components could be used in similar studies. However, extension of the current two-tone study to frequencies both above and below $1 \mathrm{kHz}$ would seem to be more interesting at this time. We would, first of all, learn whether the just-discriminable $\hat{f}(t)$ differences vary with frequency as the simple frequency $\mathrm{dL}$. Any breakdown in performance, at high frequencies, for example, would allow us to define an "existence region" for the effect and perhaps lead to further insight into pitch perception of complex tones.

\section{REFERENCES}

Cherry, E. C., \& Phillips, V. J. Some possible uses of single sideband signals in formant-tracking systems. Journal of the Acoustical Society of America, 1961, 33, 1067-1077.

Helmholtz; H. L. F. On the seneations of tone. (2nd English ed.) New York: Dover, 1954. P. 165 and Appendix XIV.

Jeffress. L. A. Beating sinusoids and pitch changes. Joumal of the Acoustical Society of America, 1968, 43, 1964.

Licklider, J. C. R. Basic correlates of the auditory stimulus. In $S$. S. Stevens (Ed.), Handbook of experimentat poychology. New York: Wiley, 1951. Pp. 1015-1021.

von Bekesy, G. Experiments in hearing. New York: Mc-Graw-Hill, 1960. P. 576.

(Received for publication September 28, 1973; accepted December 7, 1973.) 